\title{
Evaluation of EX-PRESS glaucoma implant in elderly diabetic patients after 23G vitrectomy
}

\author{
This article was published in the following Dove Press journal: \\ Clinical Interventions in Aging \\ 10 April 2017 \\ Number of times this article has been viewed
}

\author{
Anita Lyssek-Boroń' \\ Adam Wylęgała' \\ Dariusz Dobrowolski',2 \\ Ewelina Kowalczyk' \\ Katarzyna Polanowska' \\ Edward Wylęgała ${ }^{1,2}$ \\ 'Department of Ophthalmology \\ with Pediatric Unit, Trauma Center, \\ Santa Barbara Hospital, Sosnowiec, \\ ${ }^{2}$ Department of Ophthalmology, \\ Ophthalmology Clinic, Medical \\ University of Silesia, District Railway \\ Hospital, Katowice, Poland
}

Correspondence: Anita Lyssek-Boroń Department of Ophthalmology with Pediatric Unit, Trauma Center, Santa Barbara Hospital, Plac Medyków I, Sosnowiec 4I-200, Poland

$\mathrm{Tel}+4832368257 \mathrm{I}$

Fax +48 323680028

Email lyssek.boron.anita@gmail.com
Purpose: The most frequent qualifications for pars plana vitrectomy (PPV) in diabetic patients include recurrent hemorrhage into the vitreous body chamber and vitreoretinal proliferation, also with traction retinal detachment. The aim of this study was to evaluate the effectiveness of EX-PRESS implant for the treatment of secondary glaucoma in elderly diabetic patients following PPV 23G with silicon oil or SF6 gas endotamponade.

Materials and methods: This retrospective analysis comprised 18 patients (19 eyes). We applied EX-PRESS implants in 9 patients with neovascular glaucoma (NVG) and in 10 patients with non-NVG. All patients had earlier history of diabetes and vitrectomy $23 \mathrm{G}$ for diabetic complications. Intraocular pressure (IOP) was measured and compared before; 7 days; 1, 3, 6, and 12 months; and a year after the surgery.

Results: Application of the implant exhibited a lowering effect on IOP. After 1 month, 53\% of patients had IOP values beyond $22 \mathrm{mmHg}$, while $86 \%$ after 1 year.

Conclusion: The implant can be used to treat both NVG and non-NVG in diabetic patients following PPV.

Keywords: PPV, glaucoma surgery, DM, IOP

\section{Introduction}

As defined by the WHO, diabetes comprises a group of metabolic diseases characterized by hyperglycemia caused by impaired insulin effect and/or secretion. The forecasted number of patients suffering from diabetes may appear as high as 366 million in $2030 .{ }^{1}$ While more and more diabetic patients become disabled, persistent hyperglycemia ${ }^{2,3}$ brings along even more frequent ophthalmological complications, therefore indicating the surgical treatment of the condition.

The most frequent qualifications for pars plana vitrectomy (PPV) in diabetic patients include recurrent hemorrhage into the vitreous body chamber and vitreoretinal proliferation, also with traction retinal detachment. ${ }^{4}$ A continuing development of the new surgical techniques, such as with endolaser photocoagulation and with intraocular endotamponade, improves the chance for prolonging the eye functions.

Unfortunately, in advanced stages of the disease and with age of the patient, it has not been possible to eliminate the risk of glaucoma, which in diabetic patients often proves resistant to the treatment.

One of the examples is the microstent EX-PRESS shunt, made of stainless steel, which is applied to ensure a new path for the removal of the aqueous humor from between the anterior chamber and the space below the Tenon's capsule. It is $3 \mathrm{~mm}$ long and $400 \mu \mathrm{m}$ wide providing filtration through a $50 \mu \mathrm{m}$ channel. EX-PRESS appears as a promising method for long-term control of the intraocular pressure (IOP), 
particularly in patients with secondary glaucoma following vitreoretinal surgery, neovascular glaucoma (NVG), and unsuccessful trabeculectomy. 5,6

The aim of this study was to evaluate the effectiveness of EX-PRESS implant for the treatment of secondary glaucoma in diabetic patients following PPV 23G with silicon oil or SF6 gas endotamponade.

\section{Materials and methods}

This retrospective analysis comprised 18 patients (19 eyes) aged 65-81 years ( ing 11 females and 7 males with EX-PRESS (Alcon, Novartis AG, Basel, Switzerland) shunt implanted throughout 2013-2014 in St Barbara Regional Specialist Hospital in Sosnowiec. The research was approved by the Research Ethics Committee of the Silesian Medical Chamber in Katowice (resolution number ŚIL/KB/100p/17). Patient consent to review their medical records was not required by the bioethical committee due to the retrospective nature of this study and because patients' information was sufficiently anonymized. All patients had secondary glaucoma and long-term and poorly controlled diabetes. The test group included 1 patient with type 1 diabetes and 17 patients with type 2 diabetes. The patients had earlier undergone full $23 \mathrm{G}$ PPV for diabetic complications, such as vitreoretinal proliferation with hemorrhage into the vitreous body chamber (15 patients) and traction retinal detachment (4 patients). A total of 9 eyes with NVG and 10 eyes with non-NVG were included. A topical antiglaucoma treatment did not bring in any effects in all patients. Secondary non-NVG occurred after hemorrhage due to diabetic retinopathy or after PPV with silicone oil endotamponade.

All patients signed informed consent before undergoing any surgical procedure. PPV was performed using type panretinal photocoagulation endolaser photocoagulation and in the peripheral retina, within the $360^{\circ}$ range. Silicon oil endotamponade was applied in 12 patients, while SF6 was applied in 6 patients. After 1 month of procedure, silicon oil was removed. All surgeries were performed by one operator. The EX-PRESS shunt was implanted from 3 to 8 months after the PPV under the scleral flap in the upper quadrant. Following the surgery, all the patients locally received the antibiotic and steroid drops as well as mydriatics during 1 month. Despite the maximum pharmacological treatment, the indication for shunt implantation was uncontrolled IOP.

The surgery was considered successful with IOP below $22 \mathrm{mmHg}$ and above $5 \mathrm{mmHg}$, requiring no additional surgical procedures and no loss in light perception in the operated eye. The need for local drugs to lower IOP was not accepted as failure. Visual acuity was evaluated using best correction, while IOP was measured using Goldmann applanation tonometer 7 days; 1, 3, 6, and 12 months; and a year after the surgery.

\section{Statistical methods applied}

For quantitative variables, the calculations comprised mean, standard deviation, median, first quartile, and third quartile. The highest and the lowest value were also quoted. For qualitative variables, number and proportion of patients were given.

Normal distribution of IOP at subsequent observation times and differences in the pressure between particular observation times were evaluated using Shapiro-Wilk test and a histogram. The results from Shapiro-Wilk test did not show the statistical significance for all variables; however, the evaluation of the histogram did not allow to accept normal distribution of a given variable. Therefore, nonparametric tests were used for comparison.

The two-paired samples were compared using Wilcoxon signed-rank test for paired data, while Friedman test was used for multiple measurements $(>2)$. For two independent samples, Wilcoxon rank test was used. Survival evaluation was performed for "time to failure." Results were presented as Kaplan-Meier curves. Failure was defined as the pressure $\geq 22 \mathrm{mmHg}$ or $\leq 5 \mathrm{mmHg}$.

Evaluation of survival was carried out in subgroups, classified by patients with NVG and secondary diabetic non-NVG. Results of both assays were compared using log-rank test. The significance level of 0.05 was accepted for all tests (the statistically significant scores are marked in bold).

\section{Results}

Measurements of the IOP taken before the surgery (T0) as well as after 7 days and 1, 3, 6, and 12 months showed that starting with the very first month following the surgery, the pressure tended to be above the normal level (Table 1).

Table I Basic descriptive statistical values for intraocular pressure at subsequent observation times

\begin{tabular}{llllll}
\hline Variable & $\begin{array}{l}\text { Mean } \pm \text { SD } \\
(\mathbf{m m H g})\end{array}$ & Min & Median & Max & $\begin{array}{l}\text { Number } \\
\text { of patients }\end{array}$ \\
\hline T0 & $40.4 \pm 8.37$ & 26 & 40 & 57 & 19 \\
7 days & $17.8 \pm 4.95$ & 9 & 17 & 30 & 19 \\
I month & $26.2 \pm 10.01$ & 12 & 21 & 45 & 19 \\
3 months & $24.3 \pm 8.45$ & 16 & 21 & 48 & 16 \\
6 months & $24.4 \pm 9.61$ & 10 & 23 & 50 & 15 \\
I2 months & $24.6 \pm 11.79$ & 12 & 21 & 60 & 13 \\
\hline
\end{tabular}

Note: T0 - measurement before surgery.

Abbreviations: Min, minimum; Max, maximum. 


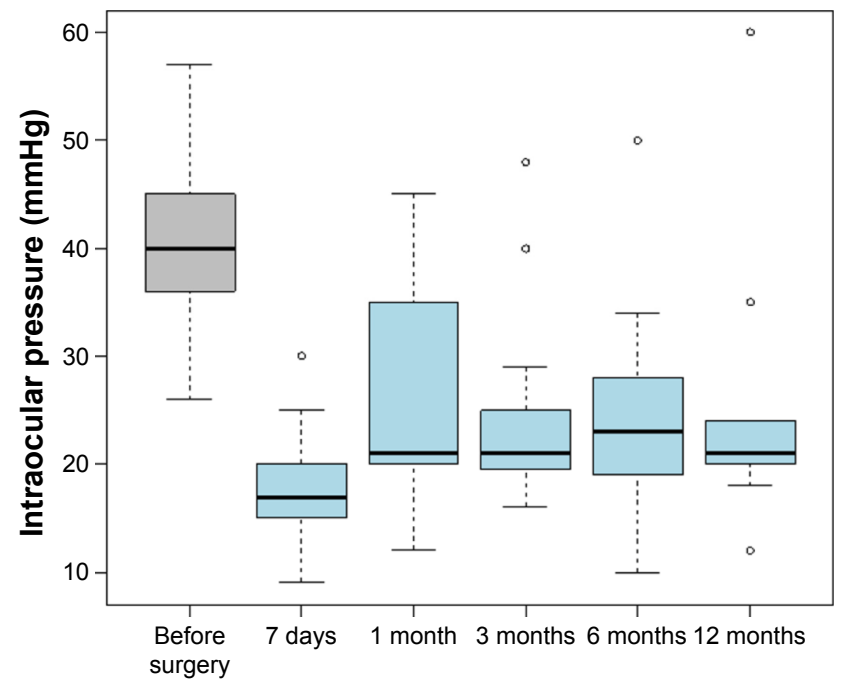

Figure I IOP before and after surgery. Abbreviation: IOP, intraocular pressure.

Each time the comparison was done showed a statistically significant score that proves that the values before the surgery are significantly higher than any moment analyzed after the surgery (Figure 1). The lowest values are 7 days after surgery, then IOP elevates and stabilizes throughout the following. Statistically significant higher results were obtained for the comparison of the 7th day after the surgery with the values recorded 1 month $(P=0.0065), 3$ months $(P=0.0085), 6$ months $(P=0.001)$, and 12 months $(P=0.0272)$ after the surgery.

The number and proportion of patients with IOP beyond the normal range $(\geq 22)$ were also assessed. Approximately 7 days after the surgery, the proportion of patients with abnormal IOP was $15.79 \%$, while the proportion after 1 month was as high as $47.37 \%$. The highest proportion of patients with abnormal IOP was recorded after 6 months following the surgery $(53.33 \%)$ (Table 2 ).

The comparison of the group of patients with NVG and those with secondary diabetic glaucoma (non-NVG) showed no statistically significant differences between the IOP value before the surgery and the scores after 7 days and 1, 3,6, and 12 months (Figure 2). After 7 days, the IOP scores for patients

Table 2 Number and percentage of patients with IOP beyond normal range $(T \geq 22)$

\begin{tabular}{lll}
\hline Time & $\begin{array}{l}\text { Number of patients } \\
\text { assessed, } \mathbf{N}\end{array}$ & $\begin{array}{l}\text { Number and percentage of } \\
\text { patients with } \mathbf{T} \geq \mathbf{2 2}, \mathbf{n} \text { (\%) }\end{array}$ \\
\hline 7 days & 19 & $3(15.79)$ \\
I month & 19 & $9(47.37)$ \\
3 months & 16 & $6(37.50)$ \\
6 months & 15 & $8(53.33)$ \\
I2 months & 13 & $6(46.15)$ \\
\hline
\end{tabular}

Abbreviation: IOP, intraocular pressure.

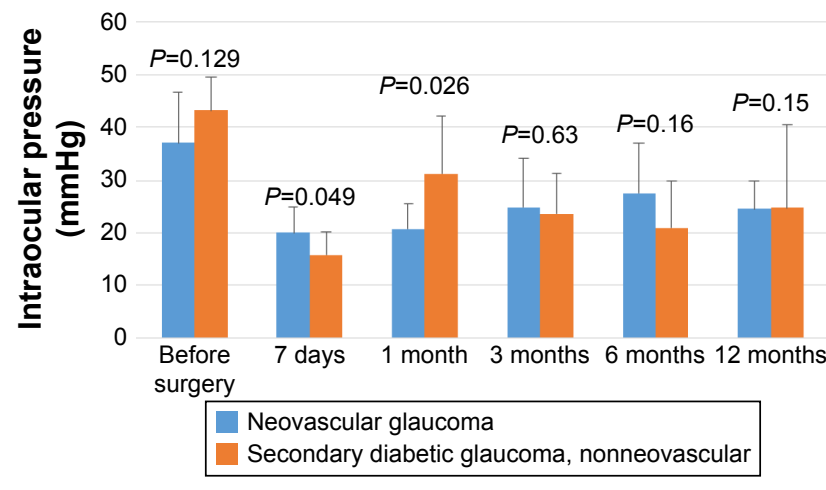

Figure 2 Comparison of intraocular pressure values in groups at subsequent observation times: patients with neovascular glaucoma and patients with secondary diabetic glaucoma, nonneovascular.

with secondary diabetic glaucoma (nonvascular) were statistically lower than the values for patients with NVG. In contrast, after 1 month, the values of patients with secondary diabetic glaucoma are significantly higher than those of patients with NVG. The mean logMAR best-corrected visual acuity (BCVA) preimplantation was 1.35 , which decreased 12 months posttreatment to 1.56 (Figure 3 ).

\section{Survival analysis}

Survival analysis was also carried out based on the data collected. Failure was defined as the occurrence of IOP beyond the normal range. The observed probability of survival without failure after 1 month following the surgery was $47 \%$. The probability of surviving 3 months without failure was only $32 \%$. The probability of surviving 6 months without failure was $21 \%$, while survival of 12 months was as little as $14 \%$ (Figure 4). The time to failure median was 1 month, and eventually, 16 cases of 19 ended up with a failure.

The survival analysis was also performed in subgroups, dividing between patients with NVG and non-NVG. The patients with NVG showed the probability of survival without



Figure 3 BCVA in all patients during the study. Abbreviation: BCVA, best-corrected visual acuity. 




Figure 4 Survival analysis results (for all patients).

failure after 1 month following the surgery to be $67 \%$. The probability of survival of 3 months without failure was $44 \%$. The probability of surviving 6 months without failure was $22 \%$, while the probability for 12 months was $0 \%$ (Figure 5).

The patients with secondary diabetic glaucoma (non$\mathrm{NVG}$ ) showed survival probability without failure after 1 month following the surgery to be $90 \%$. The probability of surviving 3 months was $20 \%$. The probability of surviving 6 months without failure was $20 \%$, while the probability for 12 months was again $20 \%$ (Figure 5).

The survival curves for patients with NVG and those with secondary diabetic glaucoma, non-NVG, were compared. No statistically significant differences were obtained for survival distribution in the evaluated groups (statistical value: $\chi^{2}=0$, degrees of freedom $=1, P=0.928$ ).

\section{Analysis of drugs}

The specification of patients with drugs shows that 1 year after the surgery, $37 \%$ of patients need no drugs and as many as $63 \%$ received some drugs. Approximately $16 \%$ take one drug, and the same proportion was observed with 2 drugs taken 1 year after the surgery, 3 drugs are taken by $25 \%$ of patients, and 4 drugs by $5 \%$ (Table 3 ). The calculations were made in



- Nonneovascular - Neovascular

Figure 5 Survival analysis results for patients with neovascular and nonneovascular glaucoma.
Table 3 Number and percentage of patients with given number of drugs at subsequent observation times

\begin{tabular}{|c|c|c|c|c|c|}
\hline \multirow{2}{*}{$\begin{array}{l}\text { Time of } \\
\text { administering } \\
\text { medication }\end{array}$} & \multicolumn{5}{|c|}{$\begin{array}{l}\text { Number and percentage of patients with } \\
\text { drugs at subsequent times, } n(\%)\end{array}$} \\
\hline & $0 \mathrm{c}$ & & gs & $3 d$ & 4 \\
\hline & & & & & \\
\hline & & & & & \\
\hline & & & & & 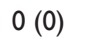 \\
\hline & & & & & \\
\hline & & $3(1$ & $4(2$ & & \\
\hline rugs after I year & $7(36.84)$ & 3 (15.79) & 3 (I5.79) & $5(26.32)$ & I (5.26) \\
\hline
\end{tabular}

the following manner: if a patient was administered 3 drugs after 3 months, then in the following lines (after 6 months and after 1 year), he is still classified as one with 3 drugs (unless the information was available on, eg, addition of another drug after a year; then the patient is included in the column " 4 drugs," in the line "after 1 year"). Patients who received no drugs had IOP values lower than $21 \mathrm{mmHg}$ (Figure 6).

\section{Discussion}

Refractory glaucoma is a disease potentially leading to loss of vision, often manifested by difficulties in control of IOP, despite high dosage medication.

The methods of glaucoma treatment such as trabeculectomy, cyclocryotherapy, or cyclophotocoagulation show low success rates, even with relevant premedication administered. The success rate 1 year after the surgery is $62.6 \%$ for trabeculectomy, ${ }^{7} 29 \%-76 \%$ for cyclocryotherapy, with major complications, ${ }^{8}$ and $52 \%-66 \%$ for cyclophotocoagulation. ${ }^{9,10}$

A novel challenge in glaucoma treatment has been EX-PRESS glaucoma filtration device, introduced throughout recent years. During the past 6 years, numerous reports were published, comparing EX-PRESS procedures to trabeculectomy ${ }^{11-13}$ as well as some reviews. ${ }^{14}$

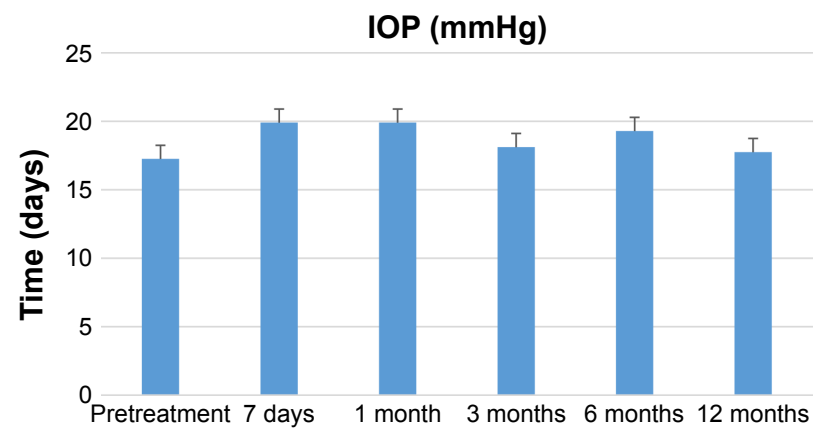

Figure 6 Intraocular pressure in patients receiving no drugs. Abbreviation: IOP, intraocular pressure. 
Despite some reports on high risk of complications, ${ }^{15}$ EX-PRESS implants ensure higher success rate and less postoperative complications scoring $70.5 \%$ and $92.9 \%$ for NVG and non-NVG, respectively. ${ }^{16}$ A recent report showed high success rate in lowering IOP after the implantation of EX-PRESS in elderly patients. ${ }^{17}$

The major risk factors for EX-PRESS implants have been identified based on the statistical analysis performed. They include non-Caucasian race, diabetes, and earlier glaucoma surgery. ${ }^{14}$ EX-PRESS implants are also accompanied by lower rate of inflammation, compared to trabeculectomy, because no tissues are actually removed such as sclerectomy and iridectomy. ${ }^{18-20}$

During our study, we applied EX-PRESS implants in patients with both NVG and non-NVG. All the patients had earlier history of diabetes and $23 \mathrm{G}$ vitrectomy due to diabetic complications.

The limitation of our study includes a relatively small sample size. The small number of patients reflects high efficacy of modern antiglaucoma drugs. The sudden decrease in IOP 7 days postsurgical treatment compared to 1 month may be attribute to lasting effects of antiglaucoma drugs, despite withdrawal. ${ }^{21}$

Application of the implant exhibited an effect in lower IOP. In all patients, the scores after the procedures were lower than the values taken before (for each of the times evaluated: 7 days and 1, 3, 6, and 12 months). Although the mean IOP values after the surgery were above $22 \mathrm{mmHg}$ with the success rate significantly lower than in patients showing no diabetes. In our opinion, the implant can also be used in elderly diabetic patients.

\section{Conclusion}

The comparison of results obtained after the implantation of EX-PRESS shunt to the values documented before the surgery showed statistically significant higher values before the surgery than after the procedures (for each of the times evaluated: 7 days and 1, 3, 6, and 12 months). The values observed 7 days after the surgery were the lowest of all. It was also noted that the scores 1, 3, 6, and 12 months after the surgery are statistically higher than the values 7 days after the surgery. We observed that the pressure was above the normal range already 1 month after the surgery. The comparison of results obtained after 1, 3, 6, and 12 months showed no statistically significant differences throughout the subsequent month after the surgery.

The analysis of survival pointed to 16 cases of failure among the total of 19. The comparison of the subgroups showed eight cases of nine ending with failure (patients with NVG). Among the patients with secondary diabetic glaucoma, 8 cases of 10 ended up with a failure.

\section{Disclosure}

The authors report no conflicts of interest in this work.

\section{References}

1. Wild S, Roglic G, Green A, Sicree R, King H. Global prevalence of diabetes: estimates for the year 2000 and projections for 2030. Diabetes Care. 2004;27(5):1047-1053.

2. Al-Shabrawey M, Zhang W, McDonald D. Diabetic retinopathy: mechanism, diagnosis, prevention, and treatment. Biomed Res Int. 2015; 2015:854593.

3. Fong DS, Aiello L, Gardner TW, et al. Retinopathy in diabetes. Diabetes Care. 2004;27(suppl 1):S84-S87.

4. Gupta V, Arevalo JF. Surgical management of diabetic retinopathy. Middle East Afr J Ophthalmol. 2013;20(4):283-292.

5. Samuelson TW, Stamper R, Gallardo M. Flow dynamics of the EX-PRESS glaucoma filtration device. US Ophthalmic Rev. 2014;7(1):39.

6. Fechtner R, de Jong L, Dahan E, Kahook M, Moster M, Goldberg I. Advances in the surgical management of glaucoma - the role of the EXPRESS $^{\circledR}$ glaucoma filtration device. Eur Ophthalmic Rev. 2012;6(2):83.

7. Takihara Y, Inatani M, Fukushima M, Iwao K, Iwao M, Tanihara H. Trabeculectomy with mitomycin $\mathrm{C}$ for neovascular glaucoma: prognostic factors for surgical failure. Am J Ophthalmol. 2009;147(5):912-918, 918.e1.

8. Benson MT, Nelson ME. Cyclocryotherapy: a review of cases over a 10-year period. Br J Ophthalmol. 1990;74(2):103-105.

9. Kosoko O, Gaasterland DE, Pollack IP, Enger CL. Long-term outcome of initial ciliary ablation with contact diode laser transscleral cyclophotocoagulation for severe glaucoma. The Diode Laser Ciliary Ablation Study Group. Ophthalmology. 1996;103(8):1294-1302.

10. Bloom PA, Tsai JC, Sharma K, et al. "Cyclodiode". Trans-scleral diode laser cyclophotocoagulation in the treatment of advanced refractory glaucoma. Ophthalmology. 1997;104(9):1508-1519; discussion 1519-1520.

11. Mermoud A. Ex-PRESS implant. Br J Ophthalmol. 2005;89(4):396-397.

12. Filippopoulos T, Rhee DJ. Novel surgical procedures in glaucoma: advances in penetrating glaucoma surgery. Curr Opin Ophthalmol. 2008;19(2):149-154.

13. Coupin A, Li Q, Riss I. Implant miniature Ex-PRESSTM placé sous un volet scléral dans la chirurgie du glaucome à angle ouvert: étude rétrospective. [Ex-PRESS miniature glaucoma implant inserted under a scleral flap in open-angle glaucoma surgery: a retrospective study]. J Fr d'ophtalmologie. 2007;30(1):18-23. French.

14. Mariotti C, Dahan E, Nicolai M, Levitz L, Bouee S. Long-term outcomes and risk factors for failure with the EX-press glaucoma drainage device. Eye (Lond). 2014;28(1):1-8.

15. Gavrić M, Gabrić N, Jagić J, Cović A. Clinical experience with ex-press mini glaucoma shunt implantation. Coll Antropol. 2011;35(suppl 2): 39-41.

16. Li Z, Zhou M, Wang W, et al. A prospective comparative study on neovascular glaucoma and non-neovascular refractory glaucoma following Ahmed glaucoma valve implantation. Chin Med J (Engl). 2014;127(8):1417-1422.

17. Huerva V, Soldevila J, Ascaso FJ, Lavilla L, Muniesa MJ, Sánchez MC. Evaluation of the Ex-PRESS $\left({ }^{\circledR}\right)$ P-50 implant under scleral flap in combined cataract and glaucoma surgery. Int J Ophthalmol. 2016;9(4): 546-550.

18. Maris PJG, Ishida K, Netland PA. Comparison of trabeculectomy with Ex-PRESS miniature glaucoma device implanted under scleral flap. J Glaucoma. 2007;16(1):14-19. 
19. de Jong LA. The Ex-PRESS glaucoma shunt versus trabeculectomy in open-angle glaucoma: a prospective randomized study. Adv Ther. 2009;26(3):336-345.

20. Marzette L, Herndon LW. A comparison of the Ex-PRESS ${ }^{\mathrm{TM}}$ mini glaucoma shunt with standard trabeculectomy in the surgical treatment of glaucoma. Ophthalmic Surg Lasers Imaging. 2011;42(6):453-459.
21. Bucci MG, Centofanti M, Oddone F, et al. Pilot study to evaluate the efficacy and safety of pneumatic trabeculoplasty in glaucoma and ocular hypertension. Eur J Ophthalmol. 2005;15(3):347-352.

\section{Publish your work in this journal}

Clinical Interventions in Aging is an international, peer-reviewed journal focusing on evidence-based reports on the value or lack thereof of treatments intended to prevent or delay the onset of maladaptive correlates of aging in human beings. This journal is indexed on PubMed Central, MedLine,
CAS, Scopus and the Elsevier Bibliographic databases. The manuscript management system is completely online and includes a very quick and fair peer-review system, which is all easy to use. Visit http://www.dovepress. com/testimonials.php to read real quotes from published authors. 